\title{
Lung Perfusion Patterns in Carcinoma of Bronchus
}

\author{
E. S. GARNETT,* M.B., M.R.C.P., M.R.C.P.ED. ; B. A. GODDARD,* B.SC., A.INST.P. ; H. S. FRASER, † M.D., D.P.H. \\ W. M. MACLEOD, $\dagger$ M.B.E., F.R.C.P.
}

Brit. med. F., 1968, 2, 209-210

Recent developments in nuclear medicine have made the measurement of ventilation or perfusion in an individual lung or lobe a relatively simple procedure. Two main techniques are currently available. In one radioactive gases are used, either inhaled or injected intravenously in solution (Dyson et al., 1960 ; West et al., 1961 ; Ball et al., 1962), the other uses isotopically labelled microemboli which are injected intravenously (Wagner et al., 1964). The latter technique was used to study lung perfusion in patients suffering from lung cancer. The microemboli were made from macroaggregates of human albumin labelled with ${ }^{131} \mathrm{I}$.

\section{Patients}

Fifty patients aged 35 to 70 years were studied; 45 were men and five were women. They were selected by chance from persons admitted to a thoracic unit with a view to surgical resection. No patients with advanced or hopelessly inoperable cancer were reviewed.

Diagnosis was established by bronchial biopsy, by sputum cell cytology, or at thoracotomy.

\section{Scanning Procedure}

${ }^{131}$ I-macroaggregates of albumin, ranging in size from 20 to $90 \mu$, were made by heating ${ }^{131}$ I human serum albumin at its isoelectric point: The size of the aggregates was checked visually by means of a haemocytometer. Approximately 300 $\mu \mathrm{C}$ of ${ }^{131} \mathrm{I}$ contained in $0.5 \mathrm{mg}$. of aggregated albumin was given intravenously after the thyroid had been blocked with iodine. The lungs were scanned immediately, the patient lying first prone and then supine, by means of a Picker Magna Scanner $V$ with a 265 -hole 5 -in. $(12.5-\mathrm{cm}$.) focus collimator.

The position of the collimator was arranged so that its plane of focus coincided with the plane between the middle and posterior thirds of the lung when the patient was prone, and the plane between the middle and anterior thirds of the lung when the patient was supine. In this way the scan obtained with the patient prone represents almost wholly the lower lobe and posterior segment of the upper lobe, while the scan obtained with the patient supine represents the upper and middle lobes.

The proportion of blood flow in each lung was measured by a modification of the method of Lopez-Majano et al. (1964). Abnormalities of local perfusion were assessed visually.

\section{Results}

We have grouped our findings under five descriptive headIngs: (1) small radiological tumour with absent lobar perfusion ; (2) perfusion of contralateral lung unexpectedly reduced; (3) patchy perfusion on both sides ; (4) bilateral reduction of perfusion with obvious lung damage; and (5) absent perfusion in disease area. \footnotetext{
- Wessex Regional Department of Nuclear Medicine, Royal South Hants
Hospital, Southampton.
} † Southampton Chest Hospital.
Group 1.-In six patients perfusion was markedly diminished in an area of lung disproportionately greater than the size of the tumour as assessed radiologically. Thus in one patient, in whom the only radiological abnormality was minimal subsegmental collapse, perfusion was absent in the whole of one lobe. In four of these patients bronchoscopy showed malignant obstruction of a main or lobar bronchus, and in two it was normal. On bronchography one of the latter patients was seen to have a block of the apico-posterior segmental branch of the left upper lobe bronchus. Angiography showed a small upper lobe artery and no filling of the superior pulmonary veins. At operation the left pulmonary artery was encircled by growth, and the superior vein was obstructed. In the sixth patient adequate bronchography showed no bronchial obstruction. Angiography showed a block of the upper lobe artery, which at operation was surrounded and invaded by an oat-cell carcinoma.

Group 2.-In nine patients perfusion of the contralateral lung was greatly diminished, though this lung was thought to be normal from clinical, radiological, and simple ventilatory studies. In seven the perfusion in the apparently "healthy" lung was less than that in the lung on the side of the neoplasm, and in two the proportion of total lung perfusion in the "healthy" lung accounted for only 35 and $37 \%$ respectively.

Group 3. Patchy Bilateral Perfusion.-In two patients the distribution of the microemboli was patchy, suggesting an irregular pattern of perfusion. On the straight radiograph there was no obvious generalized disease; but both patients gave a history of chronic bronchitis, and their ventilation tests showed moderate airways obstruction.

Group 4.- The sole patient in this group had bilaterally scarred and calcified lungs in which perfusion was irregularly distributed. The areas of decreased perfusion coincided in part with the areas of radiological damage, but in some zones perfusion was decreased where the lung appeared to be normal, while in other zones perfusion was normal where the lung appeared to be damaged.

Group 5.-In 32 patients perfusion was absent in the region of the growth. In 21 the area of decreased perfusion was confined to the area of radiological abnormality, and in 11 perfusion was decreased not only in the region of the radiological abnormality but also in the adjacent lobe. In six of these 11 patients bronchoscopy revealed an obstruction in the bronchus supplying the radiologically normal lobe, but in the remaining five the lobar and segmental bronchi were clear.

\section{Discussion}

Wholly unexpected results were obtained in approximately one-third of a group of 50 patients suffering from carcinoma of the bronchus in whom radioisotope lung scans were performed as part of a preoperative assessment programme.

In six patients (group 1) there was no perfusion in a whole lobe, though radiologically the tumour was small. In two of them the absence of perfusion was shown to be due to tumour blocking either an artery or a vein, but it is pertinent to stress that in one of these patients both bronchoscopy and bronchography were normal. Vascular blockage may have been 
the explanation for the absent lobar perfusion in the other four patients in group 1. However, it is also possible that the bronchial tumour was producing obstructive emphysema, in which case it is postulated that the intra-alveolar pressure may then have been sufficient to prevent the radioactively labelled microemboli from entering the emphysematous zone.

Wagner et al. (1965) reported four cases inf bronchogenic carcinoma in which the scan indicated obstruction of the pulmonary blood flow to a degree unsuspected from the size of the lesion on the chest radiography. These workers inflated balloons in the bronchi of anaesthetized dogs and produced a decrease in pulmonary blood flow, as shown by lung scanning. They postulated that the effect may have resulted from regional hypoxia, but in our group 1 patients the breathing of $100 \%$ oxygen from a facemask before and during the scan produced no alteration in the pattern of perfusion.

In a patient with carcinoma of the bronchus one is not usually concerned with the function of the contralateral lung, provided the overall function of the lungs is reasonable. It was therefore especially interesting to find that in patients in group 2 the perfusion in the contralateral lung was less than that in the lung already partially destroyed by tumour. Thus in almost $20 \%$ of the patients studied it is possible that pneumonectomy would have produced severe respiratory disability or even early postoperative death. Necropsy studies have not as yet been performed on any of these patients, and it can only be conjectured that the "healthy" lung has been previously damaged by thromboembolic or bronchiolar disease.

Patchy areas of decreased perfusion in both lungs in patients presumed to have emphysema, as well as carcinoma of the bronchus (group 3), or in patients with old tuberculous disease (group 4), were not unexpected. Lopez-Majano et al. (1966) described similar changes in emphysema. Nor was it surprising to find areas of decreased perfusion which coincided with the area of radiological abnormality in 21 of the 32 patients in group 5. However, in the remaining 11 patients areas of decreased perfusion were found in radiologically unaffected areas of the same lung, and in five of these there was no evidence of obstruction to lobar or segmental bronchi. As yet we are unable to explain this finding.

\section{Summary}

Lung scans were performed in 50 patients with carcinoma of the bronchus. Unexpected abnormalities of perfusion were found in one-third of them. It is therefore suggested that lung scans should be routine in the preoperative assessment of these patients.

We thank Mrs. Julie Phelps for technical assistance with the scans.

REFERENCES

Ball, W. C., Stewart, P. B., Newsham, L. G. S., and Bates, D. V. (1962). 7. clin. Invest., 41, 519 .

Dyson, N. A., Hugh-Jones, P., Newbery, G. R., Sinclair, J. D., and West, J. B. (1960). Brit. med. Ұ., 1, 231

Lopez-Majano, V., Chernick, V., Wagner, H. N., and Dutton, R. E (1964). Radiology, 83, 697.

Lopez-Majano, V., Tow, D. E., and Wagner, H. N. (1966). F. Amer. med. Ass., 197, 81.

Wagner, H. N., Sabiston, D. C., McAfee, J. G., Tow, D., and Stern, H. S. (1964). New Engl. 7. Med., 271, 377.

Wagner, H. N., Lopez-Majano, V., Tow, D. E., and Langan, J. K. (1965). Lancet, 1,344 . West, J. B., Dollery, C. T., and Hugh-Jones, P. (1961). F. clin. Invest.,
40, 1.

\title{
Percutaneous Electrical Cordotomy in Relief of Intractable Pain
}

\author{
S. LIPTON,* M.B., CH.B., F.F.A. R.C.S
}

Anterolateral cordotomy for relief of pain was first performed by Spiller and Martin (1912). It is accepted as the best available method for long-term relief despite its disadvantages. These are: a variable mortality depending on the type of case dealt with, a prolonged period of healing and convalescence in many of the patients with cancer, and a success rate which falls steadily with time (Nathan, 1963a).

The incision into the anterolateral tract is a very small one compared with the extent of the operation, and a number of percutaneous techniques have been described which produce the required lesion without surgery (Mullan et al., 1963, 1965; Rosomoff et al., 1965; Lin et al., 1966).

The percutaneous method has considerable advantages. The mortality is very small and the technique can be used on any patient up to and including the terminal state. If the required level is not attained at the first attempt, or if the level subsequently drops, the procedure is easily repeated. Most patients can be discharged four days after this type of cordotomy.

- Consultant Anaesthetist, Liverpool Regional Neurosurgical Centre, Walton Hospital, Liverpool ; Consultant to Pain Relief Clinic, Liver pool Regional Neurosurgical Centre, Walton Hospital, Liverpool.

\section{Material}

In this unit 52 patients have been treated by percutaneous electrical cordotomy: 44 had intractable pain due to cancer, 4 due to post-herpetic neuralgia, and 4 due to other nonmalignant causes. Ages varied from 31 to 80 years. All patients had been fully investigated before admission -17 were sent by neurosurgeons, 28 were referred by consultants at hospitals throughout the Liverpool region, and 7 were referred by general practitioners. Before the percutaneous cordotomy technique was available this type of patient was treated by surgical cordotomy, by intrathecal phenol, or by a long-acting paravertebral or other block. Often their medical advisers did not seek operative relief. After it was available it has been offered to all patients with intractable pain from cancer. It has also been offered to some patients having non-malignant intractable pain.

Assessment of Pain Relief.- " Relief" means freedom from pain up to the terminal stage of the disease. "Partial relief" means that there is some worth-while benefit obtained from the treatment, such as a statement from the patient to this effect or a reduction in the analgesic drugs needed. Anything not classified as a relief or partial relief is classified as a "failure." 